\title{
Energetics of walking in patients with peripheral arterial disease: a proposed functional evaluation protocol
}

\author{
Claudio MARCONI*, Guido FERRETTI†, Sara ANCHISI \\ Giovanni SCANDALEß, Antonio ANTICO\|, Gianluca IOB\|, Flavio PEINETTI\| \\ and Paolo CERRETELLI $\ddagger$
}

* Muscle Physiology and Proteome Section, I.B.F.M.-Consiglio Nazionale delle Ricerche, L.I.T.A., v. F. lli Cervi 93, I-20090

Segrate, Italy, †Department of Physiology, Medical School, University of Geneva, Geneva, Switzerland, †Department of Sciences and Biomedical Technologies, University of Milan, Milan, Italy, §Medical Angiology Unit, Research Center on Vascular Diseases, Sacco Hospital, University of Milan, Milan, Italy, and ||Division of Vascular Surgery, Aosta Regional Hospital, Aosta, Italy

\section{$\begin{array}{lllllllll}\text { A } & \text { B } & S & T & R & A & C & T\end{array}$}

\begin{abstract}
The energy cost of walking (at $\left.3.2 \mathrm{~km} \cdot \mathrm{h}^{-1}\right)$ per unit distance $\left(\mathrm{J} \cdot \mathrm{kg}^{-1} \cdot \mathrm{m}^{-1}\right)$ at gradients of $0 \%,+7 \%$, and $+12 \%$ and during a progressive test ( $2 \%$ increase in gradient every $2 \mathrm{~min}$ ), as well as the overall (aerobic plus anaerobic) net cumulative energy consumption and the corresponding maximal exercise duration were assessed in 19 patients with peripheral arterial disease (PAD) and in 13 moderately active control subjects. With a $0 \%$ gradient, the energy cost of walking was $\sim 40 \%$ greater in patients with PAD than in controls $\left(2.93 \pm 0.52\right.$ and $2.13 \pm 0.33 \mathrm{~J} \cdot \mathrm{kg}^{-1}$. $\mathrm{m}^{-1}$ respectively; $\left.P<0.0 \mathrm{I}\right)$. In contrast, at gradients of $+7 \%$ and $+12 \%$, the energy cost of walking was similar in the two groups $\left(+7 \%\right.$ : PAD, $4.15 \pm 0.74 \mathrm{~J} \cdot \mathrm{kg}^{-1} \cdot \mathrm{m}^{-1}$; controls, $4.18 \pm$ $0.54 \mathrm{~J} \cdot \mathrm{kg}^{-1} \cdot \mathrm{m}^{-1} ;+12 \%$ PAD, $5.59 \pm 1.03 \mathrm{~J} \cdot \mathrm{kg}^{-1} \cdot \mathrm{m}^{-1}$; controls, $\left.5.64 \pm 0.75 \mathrm{~J} \cdot \mathrm{kg}^{-1} \cdot \mathrm{m}^{-1}\right)$. In patients with PAD, maximal exercise duration with gradients of $0 \%,+7 \%$ and $+12 \%$ was $449 \pm 254,322 \pm 200$ and $229 \pm 150$ s respectively, whereas the net cumulative energy consumption at fatigue was almost constant at $\sim 1100 \mathrm{~J} \cdot \mathrm{kg}^{-1}$ for all gradients. The greater energy cost of walking in PAD patients compared with controls in level, but not uphill, walking is interpreted as being mainly the consequence of an altered mechanical locomotory pattern, and not of lower metabolic efficiency. For a wide range of loads, net cumulative energy consumption appears to be independent of maximal exercise duration, a finding that provides a practical criterion for assessing the degree of functional impairment of patients with PAD on metabolic grounds.
\end{abstract}

\section{INTRODUCTION}

Peripheral arterial disease (PAD) is an atherosclerotic condition that results in a decrease in oxygen availability to the locomotory muscles and, consequently, in impaired walking capacity with claudication. Moreover, the oxygen uptake $\left(\dot{V}_{\mathrm{O}_{2}}\right)$ of PAD patients when walking on the level is higher than expected [1,2]. The observed increase in the energy cost of locomotion could be due to an increase in mechanical work and/or to decreased recovery of elastic energy, i.e. to an overall decrease in biomechanical efficiency. On the other hand, a metabolic disturbance that lowers the efficiency of ATP resynthesis by oxidative phosphorylation [3-12] cannot be ruled out.

Key words: cost of locomotion, functional evaluation, level walking, peripheral arterial disease, uphill walking.

Abbreviations: $\dot{E}$, net rate of energy expenditure; $E_{\text {tot }}$, overall net cumulative energy consumption; HR, heart rate; PAD, peripheral arterial disease; $P_{\mathrm{ETCO}}$, end-tidal partial pressure of $\mathrm{CO}_{2} ; P_{\mathrm{ETO}}$, end-tidal partial pressure of $\mathrm{O}_{2} ; \dot{V}$, pulmonary ventilation; $\dot{V} \mathrm{O}_{2}$, oxygen uptake.

Correspondence: Dr Claudio Marconi (e-mail claudio.marconi@ibfm.cnr.it). 
So far, to our knowledge, no systemic measurements of the energy cost of walking on a treadmill at different speeds and inclines have been carried out in patients with PAD.

The aims of the present study were as follows. (1) To confirm whether and/or under what conditions the energy cost of walking $\left(\mathrm{J} \cdot \mathrm{kg}^{-1} \cdot \mathrm{m}^{-1}\right)$ is greater in PAD patients than in control subjects. (2) To analyse, for given exercise test protocols, the relationship between the time at which unbearable claudication pain is attained (i.e. maximal exercise duration) and the corresponding overall net cumulative energy consumption $\left(E_{\text {tot }}\right)$, in order to identify a criterion based on metabolic parameters for assessing functional impairment in patients with PAD.

\section{METHODS}

\section{Subjects}

Patients suffering from PAD with stable intermittent claudication in one or both legs were recruited from the Medical Angiology Unit of the University of Milan-Sacco Hospital, Milan, Italy, and the Aosta Regional Hospital, Aosta, Italy. Inclusion criteria were: (1) a maximal walking distance (defined as the distance that could be covered before intolerable claudication pain was attained) ranging between 120 and $360 \mathrm{~m}$, as assessed during a preliminary standard walk on a treadmill $\left(3.2 \mathrm{~km} \cdot \mathrm{h}^{-1}\right.$ on $\mathrm{a}+12 \%$ gradient); and (2) impaired performance due to claudication pain only, in the absence of symptoms such as joint pain or dyspnoea. Exclusion criteria comprised ischaemic pain at rest, ulceration or gangrene in the lower limbs, lower-extremity vascular surgery or angioplasty within the preceding 6 months, coronary artery disease, Type I (insulin-dependent) diabetes, and resting arterial blood pressure higher than 180/110 $\mathrm{mmHg}$.

The study was carried out on 19 male patients with PAD [age $65 \pm 8$ years (mean \pm S.D.); height $1.68 \pm 0.07 \mathrm{~m}$; body mass $73.1 \pm 9.2 \mathrm{~kg}$; body mass index $25.7 \pm 3.3 \mathrm{~kg} \cdot \mathrm{m}^{-2}$ ]. Control measurements were made on 13 moderately active, healthy individuals (age $58 \pm 6$ years; height $1.70 \pm 0.04 \mathrm{~m}$; body mass $73.4 \pm 11.6 \mathrm{~kg}$; body mass index $25.7 \pm 4.5 \mathrm{~kg} \cdot \mathrm{m}^{-2}$ ). All subjects gave informed consent to participate in the study, which was carried out in accordance with the principles outlined in the Declaration of Helsinki (1989) of the World Medical Association. The study was approved by the ethical committee and research review board of the institutions involved. Mean systolic arterial blood pressure was measured in the supine position in the most impaired leg and in the right and/or left arm of PAD patients. The pressure levels at rest were $101 \pm 27$ and $152 \pm 18 \mathrm{mmHg}$ in the leg and arm respectively; at the end of the preliminary standard walk they were $65 \pm 6$ and $174 \pm 5 \mathrm{mmHg}$ respectively.

\section{EXPERIMENTAL PROTOCOL}

Individual experiments were carried out in the morning, on different days and after a light meal. Room temperature and relative humidity were $20 \pm 1^{\circ} \mathrm{C}$ and $60 \pm 5 \%$ respectively.

After $30 \mathrm{~min}$ of rest (seated), the subject stood on the treadmill with his feet outside the rolling band. The speed was kept constant at $3.2 \mathrm{~km} \cdot \mathrm{h}^{-1}$. The slope was set randomly to a $0 \%,+7 \%$ or $+12 \%$ gradient. As soon as the slope had been adjusted, the subject started walking without holding the safety bar. All exercises terminated (maximal exercise duration; see Table 2) when leg pain became unbearable. No subject stopped because of dyspnoea or tachycardia. Control subjects stopped exercising after $6 \mathrm{~min}$. In addition to the above loads, both PAD patients and controls performed a graded incremental test, walking on the treadmill at $3.2 \mathrm{~km} \cdot \mathrm{h}^{-1}$ at a $0 \%$ gradient for $2 \mathrm{~min}$, followed by a $2 \%$ increase every $2 \mathrm{~min}$, to voluntary exhaustion. Controls did not reach exhaustion and were asked to stop exercising after $22 \mathrm{~min}$, when the slope was $\sim 20 \%$.

\section{Measurements}

A computerized $\mathrm{O}_{2} / \mathrm{CO}_{2}$ analyser/flowmeter combination (Vmax 229; SensorMedics) was used for breath-bybreath assessment of pulmonary ventilation $\left(\dot{V}_{\mathrm{E}}\right), \dot{V}_{\mathrm{O}_{2}}$ and $\mathrm{CO}_{2}$ output. $\dot{V}_{\mathrm{E}}$ was calculated by integration of the flow tracings recorded at the mouth of the subject by means of a highly accurate and stable mass flow sensor utilizing a pair of heated stainless steel wires to measure gas flow. Flow calibration was performed before each experiment by means of a 3-litre syringe, at different flow rates. $\dot{V} \mathrm{O}_{2}$ and $\mathrm{CO}_{2}$ output were determined by monitoring the partial pressures of $\mathrm{O}_{2}$ and $\mathrm{CO}_{2}$ continuously at the mouth of the subject throughout the respiratory cycle by means of a fast-response paramagnetic oxygen analyser and a fast-response, non-dispersive near-IR analyser respectively. Calibration of $\mathrm{O}_{2}$ and $\mathrm{CO}_{2}$ analysers was performed before each experiment using gas mixtures of known composition.

Heart rate (HR; by ECG) and arterial blood $\mathrm{O}_{2}$ saturation (by earlobe pulse oximetry; Biox II, Ohmeda) were monitored throughout the tests. At rest and at various time points $(1,3,5$ and $7 \mathrm{~min})$ during recovery after the three constant-load tests and the graded incremental exercise, $20 \mu$ l of capillary arterialized blood was taken from a preheated earlobe for determination of the blood lactate concentration, carried out using an electroenzymic method (ESAT 6661 Lactat; Eppendorf, Hamburg, Germany).

\section{Calculations}

The mean net rate of energy expenditure $(\dot{E})$ during constant-load exercise was determined as $E_{\text {tot }} /$ maximal exercise duration. The aerobic component of $E_{\text {tot }}$ was 
calculated from cumulative $\mathrm{O}_{2}$ consumption, subtracting the resting $\dot{V}_{\mathrm{O}_{2}}$ value. A caloric equivalent for $\dot{V}_{\mathrm{O}_{2}}$ of $20.9 \mathrm{~kJ} \cdot \mathrm{l}^{-1}$ was assumed. The anaerobic lactic component of $E_{\text {tot }}$ was calculated from net lactate accumulation in blood (peak lactate concentration in blood minus that at rest), assuming that a $1 \mathrm{mM}$ increment in blood lactate corresponds to $3.3 \mathrm{ml}$ of $\mathrm{O}_{2}$ spared per $\mathrm{kg}$ of body weight [13]. The anaerobic, alactic contribution was estimated from steady-state and resting $\dot{V}_{\mathrm{O}_{2}}$, assuming a monoexponential rest-to-work transient with a time constant $(\tau)$ of 23 s [14].

The net energy cost of walking per unit distance $\left(\mathrm{J} \cdot \mathrm{kg}^{-1} \cdot \mathrm{m}^{-1}\right)$ during constant-load exercise was calculated as the ratio of $\dot{E}$ to the walking speed.

\section{Statistics}

Data were expressed as means \pm S.D., and were analysed by unpaired Student's $t$ tests (two sided) and, when applicable, by one-way ANOVA. The level of significance was set at $P<0.05$.

\section{RESULTS}

Resting values of $\mathrm{HR}, \dot{V}_{\mathrm{O}_{2}}$, respiratory exchange ratio, $\dot{V}_{\mathrm{E}}$, respiratory rate, end-tidal partial pressures of $\mathrm{O}_{2}\left(\mathrm{PETO}_{2}\right)$ and $\mathrm{CO}_{2}\left(\mathrm{PETCO}_{2}\right)$, and blood lactate concentrations of PAD patients and control subjects are shown in Table 1. No significant differences were found between the two groups in any of these parameters.

The average steady-state values (means \pm S.D.) for $\mathrm{HR}, \dot{V}_{\mathrm{O}_{2}}$, respiratory exchange ratio, $\dot{V}_{\mathrm{E}}$, respiratory rate, $P_{\mathrm{ETO}_{2}}, \mathrm{PETCO}_{2}$ and blood lactate concentration during the three constant-load exercises are shown in Table 2, along with the corresponding maximal exercise duration and maximal walking distance. It appears that, when walking on the level, the $\dot{V}_{\mathrm{O}_{2}}$ of the PAD patients was $\sim 40 \%$ greater than that of controls $(11.8 \pm 2.3$ and $8.4 \pm 0.6 \mathrm{ml} \cdot \mathrm{min}^{-1} \cdot \mathrm{kg}^{-1}$ respectively; $\left.P<0.001\right)$. In contrast, no significant differences in $\dot{V}_{\mathrm{O}_{2}}$ were found between the PAD patients and controls during walking at $\mathrm{a}+7 \%$ gradient $\left(14.9 \pm 2.6\right.$ and $14.1 \pm 1.5 \mathrm{ml} \cdot \mathrm{min}^{-1}$. $\mathrm{kg}^{-1}$ respectively) or a $+12 \%$ gradient $(17.4 \pm 3.4$ and $17.8 \pm 2.0 \mathrm{ml} \cdot \mathrm{min}^{-1} \cdot \mathrm{kg}^{-1}$ respectively). Similarly, in level walking, the $\mathrm{HR}$ of PAD was $\sim 25 \%$ ( 20 beats . $\left.\min ^{-1}\right)$ higher than that of controls. The difference became smaller $(\sim 8 \%)$ with gradients of $+7 \%$ and

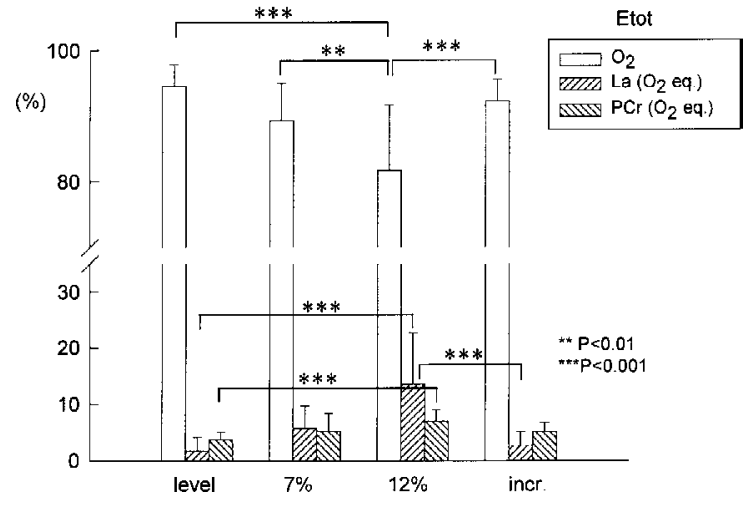

Figure I Percentage contributions of aerobic and anaerobic energy sources to the $E_{\text {tot }}$ of PAD patients at different workloads

Anaerobic energy comprises lactic (La) and alactic (phosphocreatine; $\mathrm{PCr}$ ) components, and is expressed in $\mathrm{O}_{2}$ equivalents.

$+12 \% . \dot{V} \mathrm{E}$ and blood lactate concentration were slightly greater in PAD patients than in controls. Resting arterial blood $\mathrm{O}_{2}$ saturation of both PAD and control subjects was $\sim 98 \%$, and this was not affected by exercise of any intensity (results not shown).

During the graded incremental test, PAD patients experienced severe claudication, and stopped when the average incline was $+6 \pm 4 \%$. The corresponding maximal exercise duration was $442 \pm 221 \mathrm{~s}$ and peak $\dot{V}_{\mathrm{O}_{2}}$ was $14.1 \pm 3.6 \mathrm{ml} \cdot \mathrm{min}^{-1} \cdot \mathrm{kg}^{-1}$.

The relative contributions of aerobic and anaerobic (lactic and alactic) energy sources to $E_{\text {tot }}$ of the PAD patients during the three constant-load tests and the graded incremental test are shown in Figure 1. The net total (aerobic + anaerobic) energy cost of walking per unit distance at a speed of $3.2 \mathrm{~km} \cdot \mathrm{h}^{-1}$ on the level and at gradients of $+7 \%$ and $+12 \%$ is shown in Figure 2 for both PAD patients and control subjects. Again, the energy cost of walking on the level appeared to be significantly greater $(P<0.01)$ for PAD patients than for controls $(2.93 \pm 0.52$ and $2.13 \pm 0.33$ $\mathrm{J} \cdot \mathrm{kg}^{-1} \cdot \mathrm{m}^{-1}$ respectively), and this was independent of the maximal walking distance, a practical index of severity of claudication. Indeed, the energy cost of walking on the level ranged from $3.09 \pm 0.49$ to $2.90 \pm 0.57$ $\mathrm{J} \cdot \mathrm{kg}^{-1} \cdot \mathrm{m}^{-1}$ for patients whose maximal walking distance was $\sim 250$ and $\sim 600 \mathrm{~m}$ respectively. In contrast, the net

Table I Some cardiovascular, metabolic and ventilatory variables at rest

RER, respiratory exchange ratio; $f$, respiratory rate; $\left[\mathrm{La}_{b}\right.$, blood lactate concentration.

\begin{tabular}{|c|c|c|c|c|c|c|c|c|c|}
\hline Subjects & $\begin{array}{l}\text { HR } \\
\text { (beats } \cdot \min ^{-1} \text { ) }\end{array}$ & $\begin{array}{l}\dot{V}_{0_{2}} \\
\left(I \cdot \min ^{-1}\right)\end{array}$ & $\begin{array}{l}\dot{V}_{0_{2}} \\
\left(\mathrm{ml} \cdot \min ^{-1} \cdot \mathrm{kg}^{-1}\right)\end{array}$ & RER & $\begin{array}{l}\dot{V}_{E} \\
\left(1 \cdot \min ^{-1}\right)\end{array}$ & $\begin{array}{l}f \\
\text { (breaths } \cdot \min ^{-1} \text { ) }\end{array}$ & $\begin{array}{l}\mathrm{PETO}_{2} \\
(\mathrm{mmHg})\end{array}$ & $\begin{array}{l}\text { PEtCO }_{2} \\
(\mathrm{mmHg})\end{array}$ & {$[\mathrm{La}]_{\mathrm{b}}(\mathrm{mM})$} \\
\hline PAD patients & $80 \pm 13$ & $0.30 \pm 0.08$ & $4.1 \pm 1.0$ & $0.91 \pm 0.11$ & $13.3 \pm 2.9$ & $19 \pm 3$ & $106 \pm 6$ & $30 \pm 3$ & $1.6 \pm 0.5$ \\
\hline Controls & $78 \pm 17$ & $0.28 \pm 0.07$ & $3.8 \pm 1.0$ & $0.93 \pm 0.08$ & $10.6 \pm 3.4$ & $13 \pm 2$ & $105 \pm 3$ & $35 \pm 2$ & $1.0 \pm 0.2$ \\
\hline
\end{tabular}



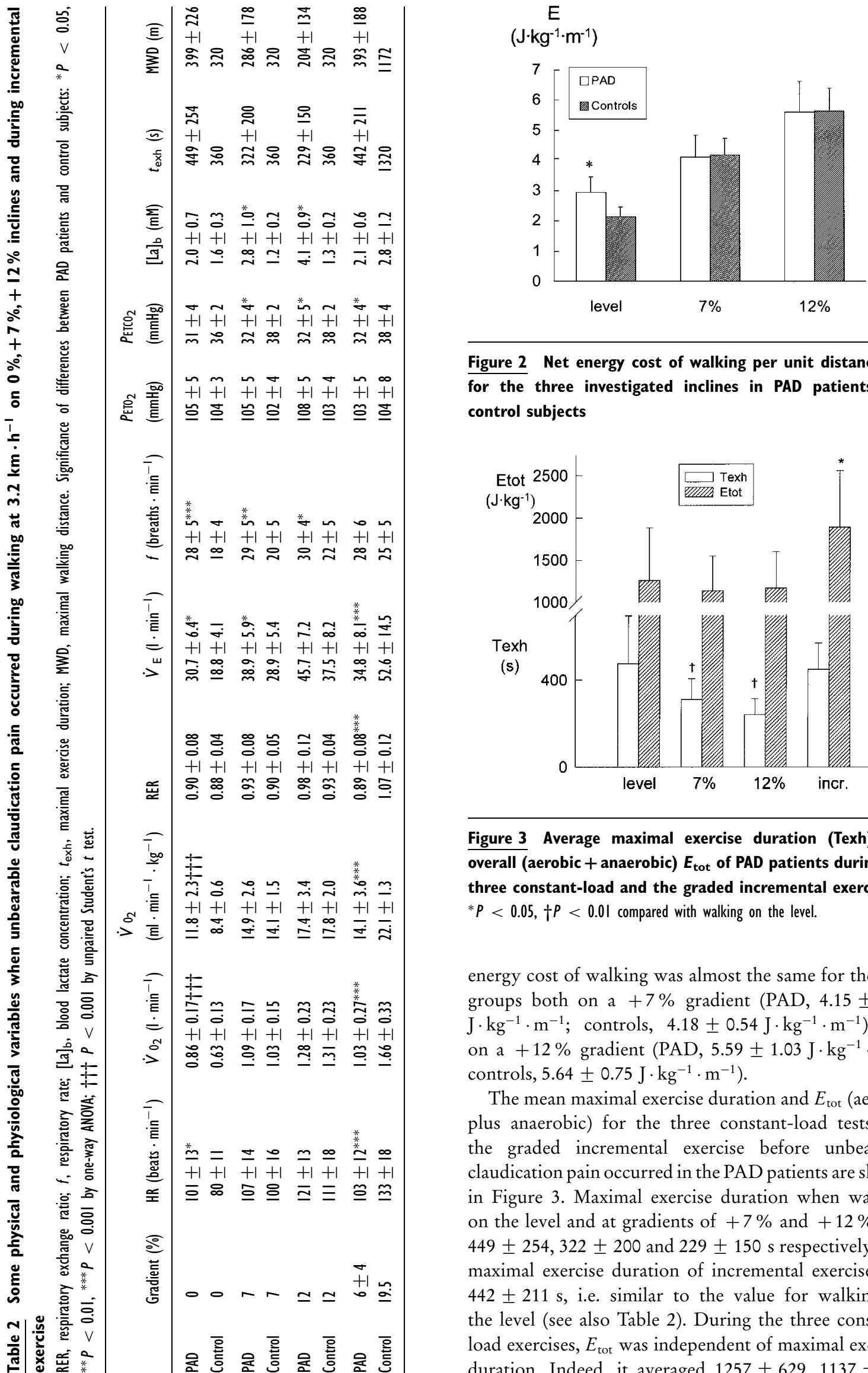

Figure 2 Net energy cost of walking per unit distance $(E)$ for the three investigated inclines in PAD patients and control subjects

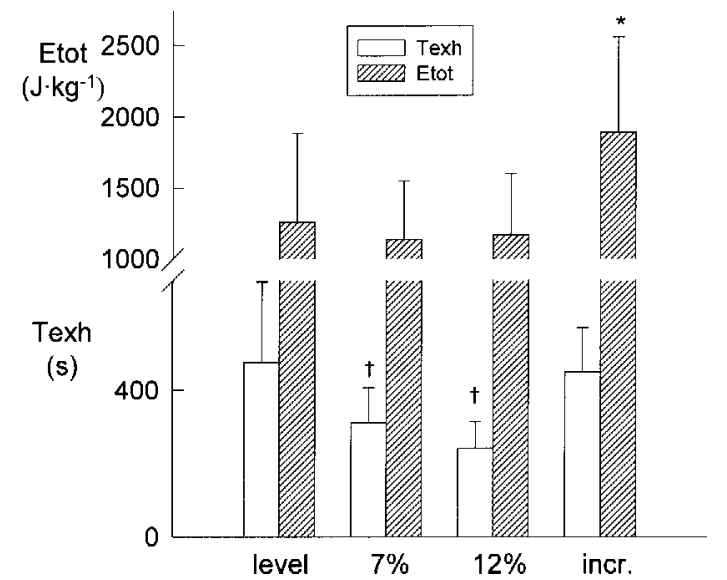

Figure 3 Average maximal exercise duration (Texh) and overall (aerobic + anaerobic) $E_{\text {tot }}$ of PAD patients during the three constant-load and the graded incremental exercises ${ }^{*} P<0.05,+P<0.01$ compared with walking on the level.

energy cost of walking was almost the same for the two groups both on a $+7 \%$ gradient (PAD, $4.15 \pm 0.74$ $\mathrm{J} \cdot \mathrm{kg}^{-1} \cdot \mathrm{m}^{-1}$; controls, $\left.4.18 \pm 0.54 \mathrm{~J} \cdot \mathrm{kg}^{-1} \cdot \mathrm{m}^{-1}\right)$ and on a $+12 \%$ gradient $\left(\mathrm{PAD}, 5.59 \pm 1.03 \mathrm{~J} \cdot \mathrm{kg}^{-1} \cdot \mathrm{m}^{-1}\right.$; controls, $\left.5.64 \pm 0.75 \mathrm{~J} \cdot \mathrm{kg}^{-1} \cdot \mathrm{m}^{-1}\right)$.

The mean maximal exercise duration and $E_{\text {tot }}$ (aerobic plus anaerobic) for the three constant-load tests and the graded incremental exercise before unbearable claudication pain occurred in the PAD patients are shown in Figure 3. Maximal exercise duration when walking on the level and at gradients of $+7 \%$ and $+12 \%$ was $449 \pm 254,322 \pm 200$ and $229 \pm 150$ s respectively. The maximal exercise duration of incremental exercise was $442 \pm 211$ s, i.e. similar to the value for walking on the level (see also Table 2). During the three constantload exercises, $E_{\text {tot }}$ was independent of maximal exercise duration. Indeed, it averaged $1257 \pm 629,1137 \pm 411$ 
and $1170 \pm 668 \mathrm{~J} \cdot \mathrm{kg}^{-1}$ at gradients of $0 \%,+7 \%$ and $+12 \%$ respectively, being significantly lower than for the graded incremental test $\left(1889 \pm 669 \mathrm{~J} \cdot \mathrm{kg}^{-1} ; P<0.05\right)$.

\section{DISCUSSION}

\section{Energy cost of locomotion}

Several authors have determined the energy cost of walking in healthy humans as a function of variables such as speed, gradient, frequency and length of stride, and age [15-20]. During level walking at $3-4 \mathrm{~km} \cdot \mathrm{h}^{-1}$, the net energy cost of walking is $\sim 2.1 \mathrm{~J} \cdot \mathrm{kg}^{-1} \cdot \mathrm{m}^{-1}$ $\left(0.5 \mathrm{kcal} \cdot \mathrm{kg}^{-1} \cdot \mathrm{km}^{-1}\right)$ [15-18]. In the present study, the net energy cost of walking of control subjects walking at $3.2 \mathrm{~km} \cdot \mathrm{h}^{-1}$ on the level was $2.1 \mathrm{~J} \cdot \mathrm{kg}^{-1} \cdot \mathrm{m}^{-1}$, and this increased when walking uphill at the same speed to 4.2 and $5.6 \mathrm{~J} \cdot \mathrm{kg}^{-1} \cdot \mathrm{m}^{-1}$ at gradients of $+7 \%$ and $+12 \%$ respectively (see Figure 2). These values are similar to those reported in the literature [15].

As shown in Figure 2, the net energy cost of walking on the level for PAD patients was $2.93 \mathrm{~J} \cdot \mathrm{kg}^{-1} \cdot \mathrm{m}^{-1}$, i.e. $\sim 40 \%$ greater than the corresponding value for control subjects; however, with steeper gradients $(+7 \%$ and $+12 \%$ ), net energy cost in PAD patients was similar to that in controls. Based on this finding, the possibility of increased energy consumption in the PAD patients for a given amount of mechanical work, i.e. of reduced metabolic efficiency, can be ruled out. It is more likely that patients with PAD are characterized by a lower mechanical efficiency of locomotion compared with control subjects, which is particularly apparent during level walking. In the latter condition, as is well known, the amount of positive mechanical work required for lifting and accelerating the body centre of mass is counterbalanced by an equal amount of negative work [21]. Since, in normal subjects, potential energy and kinetic energy are out of phase during the stride in a pendulum-like mechanism, there is a transfer of potential into kinetic energy and, as is well known, a large amount of the former is recovered [22]. Maximal energy transfer is attained in the speed range $3-5 \mathrm{~km} \cdot \mathrm{h}^{-1}$, where the work required to lift the body centre of mass at each step drops to a minimum (the so-called optimal speed [22]). The greater energy cost of walking on the level at the same speed $\left(3.2 \mathrm{~km} \cdot \mathrm{h}^{-1}\right)$ for PAD patients compared with controls may be explained by reduced recovery of potential energy through the pendulum-like mechanism and/or by additional external and/or internal work as a consequence of a change in the locomotory pattern. In fact, it was reported by some of our patients that they adjust their locomotion, particularly foot contact, to cope with claudication pain. Therefore it is likely that patients with PAD may activate different muscles during the entire stride cycle, or use the same muscle mass but with a different sequence of fibre recruitment.
Both of the above mechanisms are consistent with an increased energy cost of walking, and do not exclude each other. Indeed, preliminary data (C. Marconi, F. Saibene, L. Ardigò, M. Marzorati, M. Spelta and B. Grassi, unpublished work) confirm that total mechanical work during level walking is greater in PAD patients than in healthy age-matched control subjects, due mainly to an increased external work component. The above mechanisms could also explain the observed lowering of steady-state $\dot{V}_{\mathrm{O}_{2}}$ values in PAD patients after training, which is likely to be a consequence of improved mechanics of locomotion [23]. For any given speed, the energy cost of walking may also depend on the step frequency. However, under the present experimental conditions, the latter was essentially the same in patients as in controls, as confirmed by occasional spot determinations.

During uphill walking, it is well known that the fraction of negative work carried out by healthy individuals tends to decrease progressively with increasing incline, being close to zero above a $+15 \%$ gradient $[24,25]$. In addition, with increasing gradients, the swing of the body centre of mass during the stride becomes smaller compared with that during level walking. As a consequence, the waste of metabolic energy to lift the body centre of mass against gravity is reduced and the efficiency of uphill locomotion increases [26]. This appears to apply also to patients with PAD, whose energy cost of walking tended to resume levels similar to those of controls at gradients of $+7 \%$ and $+12 \%$. Moreover, it should be pointed out that the energy cost of walking was independent of the duration of the test exercise before unbearable claudication pain was attained. In fact, if $\dot{V}_{\mathrm{O}_{2}}$ at the time of the measurements did not reach its asymptote, the missing oxidative energy would be replaced by anaerobic sources (see Figure 1).

\section{Measurement of $E_{\text {tot }}$ : a potential tool for the assessment of functional impairment in PAD patients}

To our knowledge, there are still no standardized procedures with which to assess the walking limitations of patients with $\mathrm{PAD}$, and therefore to compare the degree of locomotory impairment among subjects undergoing different testing procedures or the effects of medical and/or rehabilitation programmes within the same patient. Several protocols differing in exercise pattern and intensity have been adopted over the last decade, with conflicting results. This is likely to be due to the variability of circulatory impairment $[27,28]$, as the latter is often distributed unevenly in the lower limbs or in different muscles of the same limb. Indeed, claudication may be the consequence either of severe ischaemia in only a small region of the muscle or of a progressive limitation of blood flow to a large muscle mass in both 
limbs. Unbearable claudication pain may occur as a consequence of a local non-sustainable rate of energy demand $(\dot{E})$, and/or of depletion of available energy (both aerobic and anaerobic; $E_{\text {tot }}$ ). Since, as is apparent from Figure 1, when walking at $3.2 \mathrm{~km} \cdot \mathrm{h}^{-1}$ on the level the contribution of anaerobic energy to $E_{\text {tot }}$ is negligible, the latter may be calculated from $\dot{V}_{\mathrm{O}_{2}}$ only. For higher gradients, allowance must be made for energy contributed by anaerobic sources.

From Figure 3, it appears that during the three constant-load protocols, maximal exercise duration became shorter with an increasing value of $\dot{E}$, whereas $E_{\text {tot }}(=$ maximal exercise duration $\cdot \dot{E})$ was rather constant, on average around $\sim 1100 \mathrm{~J} \cdot \mathrm{kg}^{-1}$. Under these circumstances, the function relating maximal exercise duration to $\dot{E}$ is an equilateral hyperbola. The rather large coefficient of variation $(\sim 35 \%)$ of the $E_{\text {tot }}$ data reflects the different degrees of functional impairment among the patients (see also Table 2). Despite this, an analysis can be attempted in order to assess the functional impairment of PAD patients on the basis of the metabolic evaluation described above.

In order to proceed with the analysis, the group of 19 PAD patients was divided into two subgroups (A and $\mathrm{B})$ according to the maximal walking distance recorded during the admission test $(\sim 140 \mathrm{~m}$ and $\sim 250 \mathrm{~m}$ for subgroups $\mathrm{A}$ and $\mathrm{B}$ respectively). The average $E_{\text {tot }}$ value for the $\mathrm{A}$ and $\mathrm{B}$ subgroups was $882 \pm 266$ and $1261 \pm 344 \mathrm{~J} \cdot \mathrm{kg}^{-1}$ respectively $(P<0.001)$. The

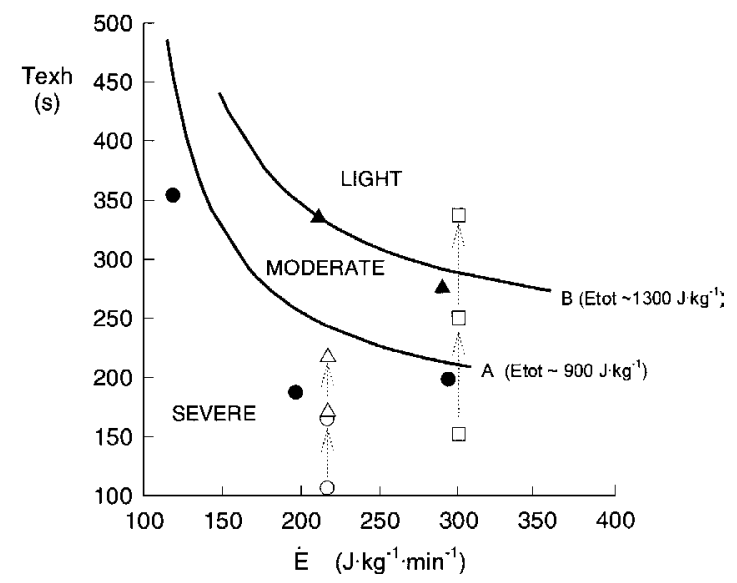

Figure 4 Maximal exercise duration (Texh) as a function of $\dot{E}$

The two hyperbolas are the best fits for $A$ and $B$ patient subgroups characterized by the indicated $E_{\text {tot }}$ values. Individual values for two typical patients with $E_{\text {tot }}$ values of $\sim 900(\mathbf{O})$ and $1300(\mathbf{\Lambda}) \mathrm{J} \cdot \mathrm{kg}^{-1}$ are also shown. Decreases in functional impairment in a group of patients after 1 and 2 months of aerobic training [29] ( $\square$ ) and in two different groups after administration of propionyl-L-carnitine [8] $(\bigcirc$ and $\triangle$ ) are shown by the vertical displacement of the corresponding symbols. Regions of severe, moderate and light functional impairment are identified. corresponding best fit of the relationship between maximal exercise duration and $\dot{E}$ is shown in Figure 4. Each of the curves, as expected, is close to a hyperbola, and the two curves describe the functional status of subgroups A and B. Within each subgroup, any given patient moves along the corresponding isopleth depending on the $\dot{E}$ value characteristic of the imposed exercise test. For practical purposes, a family of theoretical hyperbolic isopleths could be constructed for any $E_{\text {tot }}$ value within the range of interest. Thus, provided that $\dot{E}$ for any given adopted walking test (from $\dot{V}_{\mathrm{O}_{2}}$ or only from the speed and slope of the exercise protocol [15] and blood lactate accumulation [13]) and the corresponding maximal exercise duration for a given patient are known, the degree of functional impairment can be established based on the applicable isopleth. Any medical or rehabilitation treatment of the patient may result in a vertical displacement of the starting point, and therefore in a shift towards a more favourable isopleth (see Figure 4).

\section{Conclusions}

From the present study, the following conclusions may be reached. (1) The energy cost of locomotion is almost $40 \%$ greater in PAD patients than in control subjects when walking on the level at a speed of $3.2 \mathrm{~km} \cdot \mathrm{h}^{-1}$. Thus, for testing purposes, level walking in the range $3-5 \mathrm{~km} \cdot \mathrm{h}^{-1}$ should be avoided. During uphill walking, the energy cost of walking of PAD patients increases, but the differential compared with control subjects tends to disappear. It is likely that changes in the mechanics of locomotion are responsible for this finding. (2) The assessment of $E_{\text {tot }}$ of PAD patients during constant-load exercises allows the evaluatation of their walking impairment on metabolic grounds, and of the efficacy of given rehabilitation protocols. (3) As is apparent from Figure 3, PAD patients showed the greatest $E_{\text {tot }}$ values during the incremental test. Since, for rehabilitation purposes, it is generally recommended that such patients carry out the greatest possible amount of work, a graded incremental exercise may be the protocol of choice.

\section{ACKNOWLEDGMENTS}

We are grateful to all patients and control subjects who willingly collaborated in this study, to Dr W. R. Hiatt for his valuable criticisms, and to $\mathrm{Mr} \mathrm{A}$. Colombini, C. Moia and M. Pellegrini for skilled technical assistance. Financial support for this work was provided by SigmaTau, Industrie Farmaceutiche Riunite, Pomezia, Italy.

\section{REFERENCES}

1 Gardner, A. W., Skinner, J. S., Vaughn, N. R., Bryant, C. X. and Smith, L. K. (1992) Comparison of three progressive exercise protocols in peripheral vascular occlusive disease. Angiology 43, 661-671 
2 Womack, C. J., Sieminski, D. J., Katzel, L. I., Yataco, A. and Gardner, A. W. (1997) Oxygen uptake during constant-intensity exercise in patients with peripheral arterial occlusive disease. Vasc. Med. 2, 174-178

3 Zatina M. A., Berkowitz, H. D., Gross, G. M., Maris, J. M. and Chance, B. (1986) ${ }^{31} \mathrm{P}$ nuclear magnetic resonance spectroscopy: noninvasive biochemical analysis of the ischemic extremity. J. Vasc. Surg. 3, 411-420

4 Hiatt, W. R., Nawaz, D. and Brass, E. P. (1987) Carnitine metabolism during exercise in patients with peripheral vascular disease. J. Appl. Physiol. 62, 2383-2387

5 Hiatt, W. R., Wolfel, E. E., Regensteiner, J. G. and Brass, E. P. (1992) Skeletal muscle carnitine metabolism in patients with unilateral peripheral arterial disease. J. Appl. Physiol. 73, 346-353

6 Regensteiner, J. G., Wolfel, E. E., Brass, E. P. et al. (1993) Chronic changes in skeletal muscle histology and function in peripheral arterial disease. Circulation 87, 413-421

7 Kemp, J. K., Hands, L. J., Ramaswami, G. et al. (1995) Calf muscle mitochondrial and glycogenolytic ATP synthesis in patients with claudication due to peripheral vascular disease analysed using ${ }^{31} \mathrm{P}$ magnetic resonance spectroscopy. Clin. Sci. 89, 581-590

8 Taylor, D. J., Amato, A., Hands, L. J. et al. (1996) Changes in energy metabolism of calf muscle in patients with intermittent claudication assessed by ${ }^{31} \mathrm{P}$ magnetic resonance spectroscopy: a phase II open study. Vasc. Med. $1,241-245$

9 Bauer, T. A., Regensteiner, J. G., Brass, E. P. and Hiatt, W. R. (1999) Oxygen uptake kinetics during exercise are slowed in patients with peripheral arterial disease. J. Appl. Physiol. 87, 809-816

10 Bhat, H. K., Hiatt, W. R., Hoppel, C. L. and Brass, E. P. (1999) Skeletal muscle mitochondrial DNA injury in patients with unilateral peripheral arterial disease. Circulation 99, 807-812

11 Pipinos, I. I., Shepard, A. D., Anagnostopoulos, P. V., Katsamouris, A. and Boska, M. D. (2000) Phosphorus 31 nuclear magnetic resonance spectroscopy suggests a mitochondrial defect in claudicating skeletal muscle. J. Vasc. Surg. 31, 944-952

12 Brass, E. P., Hiatt, W. R., Gardner, A. W. and Hoppel, C. L. (2001) Decreased NADH dehydrogenase and ubiquinol-cytochrome c oxidoreductase in peripheral arterial disease. Am. J. Physiol. Heart Circ. Physiol. 280, H603-H609

13 Margaria, R., Aghemo, P. and Sassi, G. (1971) Lactic acid production in supramaximal exercise. Pflügers Arch. 326, 152-161
14 Binzoni, T., Ferretti, G., Schenker, K. and Cerretelli, P. (1992) Phosphocreatine hydrolysis by ${ }^{31}$ P-NMR at the onset of constant-load exercise in man. J. Appl. Physiol. $73,1644-1649$

15 Margaria, R. (1938) Sulla fisiologia, e specialmente sul consumo energetico, della marcia e della corsa a varie velocità ed inclinazioni del terreno. Atti Acc. Naz. Lincei 7,299-368

16 Bobbert, A. C. (1960) Energy expenditure in level and grade walking. J. Appl. Physiol. 15, 1015-1021

17 Cotes, J. E. and Meade, F. (1960) The energy expenditure and mechanical energy demand in walking. Ergonomics 3, 97-119

18 Dill, D. B. (1965) Oxygen used in horizontal and grade walking and running on the treadmill. J. Appl. Physiol. 20, 19-22

19 Cavagna, G. A., Franzetti, P. and Fuchimoto, T. (1983) The mechanics of walking in children. J. Physiol. (Cambridge, U.K.) 343, 323-339

20 Minetti, A. E., Capelli, C., Zamparo, P., di Prampero, P. E. and Saibene, F. (1995) Effects of stride frequency on mechanical power and energy expenditure of walking. Med. Sci. Sports Exercise 27, 1194-1202

21 Cavagna, G. A. and Margaria, R. (1966) Mechanics of walking. J. Appl. Physiol. 21, 271-278

22 Cavagna, G. A., Thys, H. and Zamboni, A. (1976) The sources of external work in level walking and running. J. Physiol. (Cambridge, U.K.) 262, 639-657

23 Hiatt, W. R., Wolfel, E. E., Meier, R. H. and Regensteiner, J. G. (1994) Superiority of treadmill walking exercise versus strength training for patients with peripheral arterial disease. Implications for the mechanism of the training response. Circulation 90, 1866-1874

24 Margaria, R. (1966) Positive and negative work performances and their efficiencies in human locomotion. Int. Z. Angew. Physiol. Einschl. Arbeitphysiol. 25,339-351

25 Minetti, A. E., Ardigò, L. P. and Saibene, F. (1993) Mechanical determinants of gradient walking energetics in man. J. Physiol. (Cambridge, U.K.) 471, 725-735

26 Margaria, R., Cerretelli, P., Aghemo, P. and Sassi, G. (1963) Energy cost of running. J. Appl. Physiol. 18, 367-370

27 Hiatt, W. R., Hirsch, A. T., Regensteiner, J. G. and Brass, E. P. (1995) Vascular Clinical Trialists: Clinical trials for claudication. Assessment of exercise performance, functional status, and clinical end points. Circulation 92, 614-621

28 Regensteiner, J. G. and Hiatt, W. R. (1995) Exercise rehabilitation for patients with peripheral arterial disease. Med. Sports Sci. Rev. 23, 1-24

29 Ernst, E. E. W. and Matrai, A. (1987) Intermittent claudication, exercise, and blood rheology. Circulation 76, 1110-1114 